\section{Óbices da governança pública e sua influência sobre a atenção à saúde bucal no Estado de Santa Catarina, Brasil}

\author{
Obstacles to public governance and their \\ influence on oral healthcare in the state of Santa \\ Catarina, Brazil
}

\author{
Obstáculos de la gobernanza pública y su \\ influencia sobre la atención en la salud bucal en \\ el estado de Santa Catarina, Brasil
}

Heloisa Godoi 1

Renata Goulart Castro 1

José Luís Guedes dos Santos 1

Samuel Jorge Moyses 2

Ana Lúcia Schaefer Ferreira de Mello 1

doi: 10.1590/0102-311X00184719

\section{Resumo}

Objetivou-se analisar a governança pública empreendida na rede regionalizada de atenção à saúde em Santa Catarina, Brasil, e sua influência sobre a tomada de decisão quanto à estruturação da atenção à saúde bucal. Estudo exploratório-analítico, com abordagem qualitativa e sustentação metodológica na Teoria Fundamentada nos Dados. Realizaram-se 30 entrevistas em profundidade com gestores das Comissões Intergestores Regional (CIR) e Bipartite (CIB), da Secretaria Estadual de Saúde, representantes do Conselho Estadual de Saúde e gestores de serviços especializados em saúde bucal, em dois grupos amostrais, além de observação não participante em reuniões da CIB. Após análise comparativa dos dados, formulou-se o modelo teórico cuja categorial central foi expressa sob título Influência das Falhas da Governança sobre a Rede de Atenção à Saúde Bucal: A Expansão Errante de Serviços por Indução Vertical e Ratificação do Caráter Periférico Dado à Saúde Bucal. A governança empreendida no sistema de saúde catarinense apresenta estruturas formais $e$ de apoio bem estabelecidas, como a consolidação das CIR. No entanto, identificaram-se fragilidades as quais revelam problemas na legitimidade do sistema de governança. Perpetua-se a posição periférica dada à estruturação da atenção à saúde bucal em rede e à expansão errante dos serviços, caracterizada por indução vertical e sem o reconhecimento contextualizado dos agravos em saúde bucal como guia para o planejamento dos serviços. Há a necessidade de maior conscientização pública e inclusão de profissionais e gestores com espírito de advocacy para propugnar um novo protagonismo da atenção à saúde bucal nas políticas públicas de saúde.

Administração de Serviços de Saúde; Regionalização; Saúde Bucal; Direção e Governança do Setor de Saúde

\section{Correspondência}

H. Godoi

Rua Douglas Seabra Levier 163, Florianópolis, SC 88040-410, Brasil.

heloisagodoi@gmail.com

1 Universidade Federal de Santa Catarina, Florianópolis, Brasil. 2 Pontifícia Universidade Católica do Paraná, Curitiba, Brasil. 


\section{Introdução}

O conceito de governança se origina de diferentes disciplinas e é multidimensional ${ }^{1}$. Por uma perspectiva analítica 2 e uma visão mais ampla, a governança pode ser observada em qualquer sociedade por meio de seus sistemas de tomada de decisão e resolução de conflitos e engloba os processos, as estruturas e os atores pelos quais as decisões são tomadas e como eles podem ser implementados 1,3. Aplicada à organização de sistemas de saúde, compreende a ação conjunta da sociedade civil e do Estado na busca por soluções e resultados para problemas comuns, buscando construir consensos que levem à formulação de políticas que respondam equilibradamente ao que a sociedade espera do governo 4.

Pesquisas no contexto internacional 2,3 e nacional 4,5,6 têm sido realizadas para identificar e compreender processos de governança com intuito de contribuir para arranjos mais avançados. Nesse sentido, diversos países empregam a descentralização do sistema de saúde para níveis locais ou regionais de governo 3,7,8. A habilidade desses diferentes níveis, que possuem suas funções e poderes, de interação coletiva em uma governança multinível determina se os resultados serão bons ou não para os sistemas de saúde e para a população ${ }^{3}$. Assim, governança em saúde tem se tornado um tema frequente na agenda do desenvolvimento locorregional 1,3 e em pesquisas sobre estruturação de sistemas de saúde. No Brasil, tem contribuído para consolidação do processo de regionalização instituído no Sistema Único de Saúde (SUS) 6,9.

O processo de descentralização da gestão do sistema de saúde brasileiro trouxe maior democratização, expansão da cobertura assistencial e inserção dos municípios como responsáveis pela condução e efetivação das políticas de saúde em nível local 10,11. Nesse processo, a regionalização se caracteriza como uma complexa tarefa em um contexto federativo em que cada esfera administrativa apresenta responsabilidades para com a construção do sistema de saúde 10 . O contexto regional é constituído como uma arena política de disputas de interesses, com o protagonismo de distintos atores 5. Assim, há a necessidade de constituição de espaços interinstitucionais por meio dos quais se estabeleçam alianças e se efetivem o planejamento, a programação e a regulação das ações e serviços de saúde, garantindo, de forma mais racional, o acesso da população a todos os níveis de atenção 5,11,12. Porém, as iniquidades encontradas entre os municípios brasileiros, em sua maioria de pequeno porte populacional, a intensa fragmentação do sistema e as disputas por recursos federais dificultaram a conformação de redes intermunicipais e adiaram o processo de regionalização do sistema de saúde brasileiro e sua capacidade resolutiva 11,12. A heterogeneidade na capacidade de provisão de serviços de saúde reforça a necessidade de os municípios se articularem de forma solidária em uma rede integrada e regionalizada de atenção 10 .

Tais estruturas apresentam papel estratégico no processo de governança pública ao criarem um canal permanente e contínuo de negociação e decisão entre os municípios e o Estado, favorecendo as relações de cooperação entre os stakeholders envolvidos 12 . Estudar a governança exercida em sistemas de saúde é importante para entender como as instituições se organizam, gerenciam conflitos e tomam decisões coletivas. A maneira como a governança é exercida afeta a tomada de decisão e quais políticas serão escolhidas e como serão executadas 3 .

No âmbito dos problemas de saúde pública, as desordens bucais possuem grande destaque 13,14 caso se leve em consideração a carga de doenças e o impacto que elas têm sobre o indivíduo e a sociedade, em termos de morbidade e custos 15 . O Brasil é um dos poucos países com sistemas universais de saúde que incluem a atenção à saúde bucal como direito de seus cidadãos 16 .

A esfera pública expandiu os serviços de atenção à saúde bucal após instituição das diretrizes da Política Nacional de Saúde Bucal (PNSB) 16. Entretanto, persiste o desafio de construir uma rede integrada de atenção à saúde bucal coerente com os princípios do SUS 16,17 que contribua para superar as constantes desigualdades em saúde bucal tanto na distribuição dos agravos, quanto no acesso e utilização dos serviços $16,18,1$.

A organização de uma rede de atenção à saúde que vise articular e integrar serviços e municípios, precisa estar ancorada em um sistema de governança operante, consolidado, participativo, presente em espaços que possibilitem a negociação, pactuação e geração de consensos objetivando a melhor articulação dos pontos de atenção à saúde bucal no âmbito regional 20,21. Estudos realizados no Estado de Santa Catarina, Brasil, com foco sobre a estruturação da atenção à saúde bucal em municípios de 
grande porte 17 e com análise dos processos decisórios realizados pela instância de governança estadual conhecida como Comissão Intergestores Bipartite (CIB) 17,21, mostraram que houve expansão nos serviços de saúde bucal como efeito do processo de governança vigente. Contudo, a articulação e integração dos serviços aparentemente continuam sendo um óbice a ser superado. Por conseguinte, o presente estudo teve como objetivo compreender a governança pública empreendida na rede regionalizada de atenção à saúde e sua influência sobre a tomada de decisão e estruturação da atenção à saúde bucal.

\section{Método}

\section{Desenho do estudo}

Trata-se de um estudo de natureza exploratório-descritiva e analítica, com abordagem qualitativa tanto para coleta quanto para análise dos dados. Seguiu o percurso metodológico da Teoria Fundamentada nos Dados (TFD) 22,23. Esta pesquisa seguiu as recomendações da Resolução $n^{\circ}$ 466, de 2012, do Conselho Nacional de Saúde. O projeto foi aprovado pelo Comitê de Ética em Pesquisa da instituição dos pesquisadores (protocolo no 1.355.582) e todos os participantes receberam e assinaram Termo de Consentimento Livre e Esclarecido.

\section{Local do estudo}

O estudo foi conduzido no Estado de Santa Catarina, localizado na Região Sul do Brasil, com população de aproximadamente 7 milhões de habitantes (Instituto Brasileiro de Geografia e Estatística. https://cidades.ibge.gov.br, acessado em 01/Mai/2017). O estado conta com 295 municípios e mantém 36 Agências de Desenvolvimento Regional (ADR) que incorporam, entre outras gerências, a Gerência de Saúde (GERSA) que atua como órgão gestor, representante do nível estadual de governo no processo de descentralização administrativa. Além dessa divisão administrativa regional, o Estado está dividido em 16 Regiões de Saúde (RS) e nove Macrorregiões de Saúde que foram discutidas e acordadas na CIB.

A atenção à saúde bucal, no Estado de Santa Catarina, conta com uma estrutura de serviços de atenção básica e especializados. Em setembro de 2019, o estado apresentava uma cobertura estimada da população atendida por serviços de saúde bucal na atenção básica de 58,78\%. Desses, 1.038 equipes de saúde bucal estavam inseridas na estratégia de saúde da família. Para realização de serviços especializados, o estado possui 49 Centros de Especialidades Odontológicas (CEO) que ofertam atendimento para 274 municípios. Quarenta e quatro CEO recebem incentivo estadual para prestação de serviços regionalizados; 3 CEO em implantação; 164 municípios credenciados para implantação de Laboratório Regional de Prótese Dentária. Além desses serviços, apresenta 8 hospitais pactuados para atendimento odontológico hospitalar para pessoas portadoras de necessidades especiais, mas ainda concentrados em região litorânea e insuficientes para a demanda 24.

No presente estudo, iniciou-se a coleta de dados pelas instâncias de governança estabelecidas por meio de leis e decretos ministeriais, conhecidas como Comissões Intergestores Bipartite (CIB) e Comissões Intergestores Regionais (CIR), Conselho Estadual de Saúde (CES) e Secretaria Estadual de Saúde (SES). O Estado possui 16 CIR, uma para cada RS. Elas desempenham importante papel para planejar, organizar, gerir e executar os serviços públicos de saúde. Além disso, a pesquisa também envolveu os serviços especializados e regionalizados de saúde bucal (CEO).

\section{Participantes do estudo}

Foram incluídos intencionalmente os atores-chave no processo de governança do sistema público de saúde no estado e na constituição da rede regionalizada de atenção à saúde (e de saúde bucal). $\mathrm{O}$ conceito de amostragem teórica 22,23,25 foi utilizado para composição de dois grupos de participantes.

No primeiro, foram convidados a participar 14 secretários de saúde, coordenadores das CIR, representando 264 municípios do estado. Dois coordenadores de CIR não responderam aos contatos 
e solicitações dos pesquisadores e foram considerados perdas amostrais. Além dos representantes das CIR, compuseram esse grupo: um membro da equipe de coordenação da CIB, um representante da área de controle e avaliação da atenção básica e um representante da área de planejamento e regulação da SES, que participavam ativamente nas câmaras técnicas instituídas na CIB, e um representante da coordenação de saúde bucal do estado. Como representantes da sociedade civil, foram incluídos três representantes dos usuários, dois representantes dos profissionais, um representante dos gestores e um dos prestadores de serviços. Dessa forma, o primeiro grupo contou com 25 participantes. O segundo grupo foi composto por quatro coordenadores de serviços especializados e regionalizados em saúde bucal, os quais possuíam experiência sobre a regionalização de serviços. Também foi realizada uma segunda entrevista com o representante da coordenação de saúde bucal do estado.

\section{Coleta dos dados}

Os dados foram coletados por meio de entrevistas individuais em profundidade. As entrevistas do primeiro grupo ocorreram inicialmente entre os meses de outubro de 2016 e março de 2017 e, em um segundo momento, em outubro de 2017. As entrevistas do segundo grupo ocorreram em outubro de 2017. A composição desses grupos ocorreu em razão de sua relevância teórica para o seguimento do desenvolvimento das categorias emergentes, propriedades, hipóteses e integração da teoria. A saturação teórica, o ponto em que novos dados não mais promovem insights teóricos, foi utilizada como critério para encerrar a coleta dos dados 25,26. Portanto, a pesquisa contou com 29 participantes, tendo sido realizadas 30 entrevistas no total.

As entrevistas foram conduzidas pela pesquisadora principal, presencialmente, em local determinado pelo participante, conforme sua disponibilidade, ou por meio de contato telefônico. Utilizou-se, como ferramenta de auxílio, um roteiro previamente elaborado com base no objetivo traçado para a pesquisa. Teve-se como base o Decreto no 7.508/2011 27, que regulamenta a Lei Orgânica da Saúde e dispõe sobre a organização do SUS com ênfase na regionalização e constituição da rede de atenção à saúde, bem como trata de estruturas e mecanismos de governança.

Além das entrevistas em profundidade, houve participação da pesquisadora principal em duas reuniões da CIB, em agosto e outubro de 2016, sendo uma delas a reunião ampliada, com participação maciça dos secretários municipais de saúde. Utilizou-se a técnica de observação não participante, o que gerou notas de campo manuscritas 23.

As entrevistas foram gravadas em dispositivo eletrônico e, junto com as notas, transcritas e codificadas, com uso do Microsoft Word (https://products.office.com/) e inseridas no software NVivo 10 (https://www.qsinternational.com/nvivo/home) para organização e categorização dos dados e elaboração de memorandos.

\section{Análise dos dados}

A análise ocorreu concomitantemente à coleta dos dados. Na TFD, essas etapas se sobrepõem para que possa haver a conceituação dos dados - codificação e categorização - e então emergir o padrão latente do comportamento social que explica o problema em estudo. A conceituação, valendo-se do conteúdo das entrevistas, bem como das notas de campo registradas durante as reuniões da CIB, ocorreu por meio de análise comparativa 23,25. Foram elaborados memorandos durante todo o processo de análise dos dados, com informações sobre os dados e conexões entre as categorias que contribuíram para o desenvolvimento do modelo teórico. Adotaram-se também diagramas para representar as relações entre códigos, conceitos e categorias emergentes 23 .

Após identificação das categorias principais do modelo, com seus respectivos conteúdos, procedeu-se da codificação teórica à compreensão dos problemas ou falhas potenciais e reais na governança que podem causar insucessos na política de saúde 3. Os códigos teóricos foram selecionados pelo quadro para análise da governança de sistemas de saúde proposto por Greer et al. 3 (Quadro 1). Na TFD, a etapa da codificação teórica permite que os códigos se relacionem uns com os outros, como hipóteses, para serem integrados à teoria 23,25 .

A formulação do modelo teórico sobre a governança empreendida na rede de atenção à saúde bucal ocorreu a partir das conexões teóricas entre as categorias, por meio da ordenação e integração 


\section{Quadro 1}

Atributos de governança conforme ferramenta de análise da governança em sistemas de saúde proposto por Greer et al. 3. Santa Catarina, Brasil, 2018.

\begin{tabular}{|c|c|}
\hline Atributo & Conceito \\
\hline Transparência & $\begin{array}{l}\text { Divulgação clara e útil de dados e informações sobre o processo de tomada de } \\
\text { decisão para o público e especialistas, os quais poderão fiscalizar, avaliar e desafiar os } \\
\text { responsáveis por gerir e formular as políticas. Resulta em maior confiança para o sistema } \\
\text { de saúde. }\end{array}$ \\
\hline Accountability & $\begin{array}{c}\text { Prestação de contas entre agências, à comunidade, aos stakeholders e aos mecanismos } \\
\text { de contratos, financiamento e políticas que criam responsabilização clara entre os entes } \\
\text { e instituições. Política de conflitos de interesses que possa evitar decisões motivadas por } \\
\text { interesses pessoais. }\end{array}$ \\
\hline Participação & $\begin{array}{l}\text { Garantia de participação estruturada e significativa de stakeholders em fóruns, com } \\
\text { mecanismos claros de escolha, que possibilite acesso à tomada de decisão e ao } \\
\text { compartilhamento do poder por todos os interessados e envolvidos, contribuindo para } \\
\text { maior legitimidade das decisões políticas e implementações. }\end{array}$ \\
\hline Integridade & $\begin{array}{l}\text { Definição clara das funções e do papel organizacional. Atores envolvido aptos a } \\
\text { compreender e predizer como a instituição realiza a tomada de decisão e possuir uma } \\
\text { trajetória de carreira sólida por meio de uma política de recursos humanos que evite } \\
\text { intervenções políticas. }\end{array}$ \\
\hline Capacidade Política & $\begin{array}{l}\text { Possuir e manter pessoal capacitado para construir políticas alinhadas com recursos } \\
\text { oferecidos e capazes de atingir os objetivos do sistema; possuir mecanismos para } \\
\text { incorporar conselhos especializados para formulação política. Experiência e atuação de } \\
\text { forma integrada dentro da organização. }\end{array}$ \\
\hline
\end{tabular}

Fonte: adaptado de Greer et al. 3.

do conteúdo, até haver o momento da saturação teórica 26 e o desenvolvimento da categoria central. A categoria central incorporou as demais categorias e, pelo estabelecimento da inter-relação entre elas, explica como o problema principal do estudo é abordado 23.

\section{Resultados}

Os entrevistados foram em sua maioria homens, com mais de 40 anos e nível superior completo. Porém, com tempo de atuação em sua função, relacionada aos fóruns de discussão/deliberação, inferior a dois anos. Todos os gestores de saúde, em nível municipal, apresentavam cargo por indicação política. Em nível estadual, havia ocorrido mudanças recentes na estrutura organizacional. Os representantes do Conselho Estadual de Saúde foram os que apresentaram maior tempo de atuação em sua função como conselheiros. Os coordenadores de serviços regionalizados de saúde bucal eram homens, tendo em média 45 anos, formação em Odontologia e título de pós-graduação correlata à área.

O modelo teórico fundamentado nos dados tem como categoria central: Influência das Falhas da Governança sobre a Rede de Atenção à Saúde Bucal: A Expansão Errante de Serviços por Indução Vertical e Ratificação do Caráter Periférico dado à Saúde Bucal (Quadro 2). A governança objeto deste estudo, multinível, e com complexidades inerentes às relações intergovernamentais, apresenta conflitos característicos do processo de descentralização em curso no país e possui falhas. Tal modelo explicita o modo como, pelas falhas nos seus atributos, a governança exerce influência indesejada sobre a tomada de decisões, com consequências negativas para estruturação da atenção à saúde bucal, dificultando o aprimoramento dos seus processos. 
Quadro 2

Categoria central e demais categorias do modelo teórico. Santa Catarina, Brasil, 2018.

Categoria central

Influência das falhas da governança sobre a rede de atenção à saúde bucal: expansão errante de serviços por indução vertical e ratificação do caráter periférico dado à saúde bucal.

\begin{tabular}{|c|c|c|}
\hline Categorias & Subcategorias & Exemplos de dados brutos \\
\hline $\begin{array}{l}\text { Estabelecimento de } \\
\text { estruturas formais } \\
\text { de governança }\end{array}$ & $\begin{array}{c}\text { • Estruturas, } \\
\text { mecanismos e } \\
\text { instrumentos } \\
\text { de governança } \\
\text { formalmente } \\
\text { estabelecidos, } \\
\text { destacando-se atuação } \\
\text { da CIR. } \\
\text { • Estruturas de apoio } \\
\text { técnico e interferências } \\
\text { de agentes dos poderes } \\
\text { executivo, legislativo e } \\
\text { judiciário identificadas } \\
\text { (crescente judicialização } \\
\text { da saúde). }\end{array}$ & 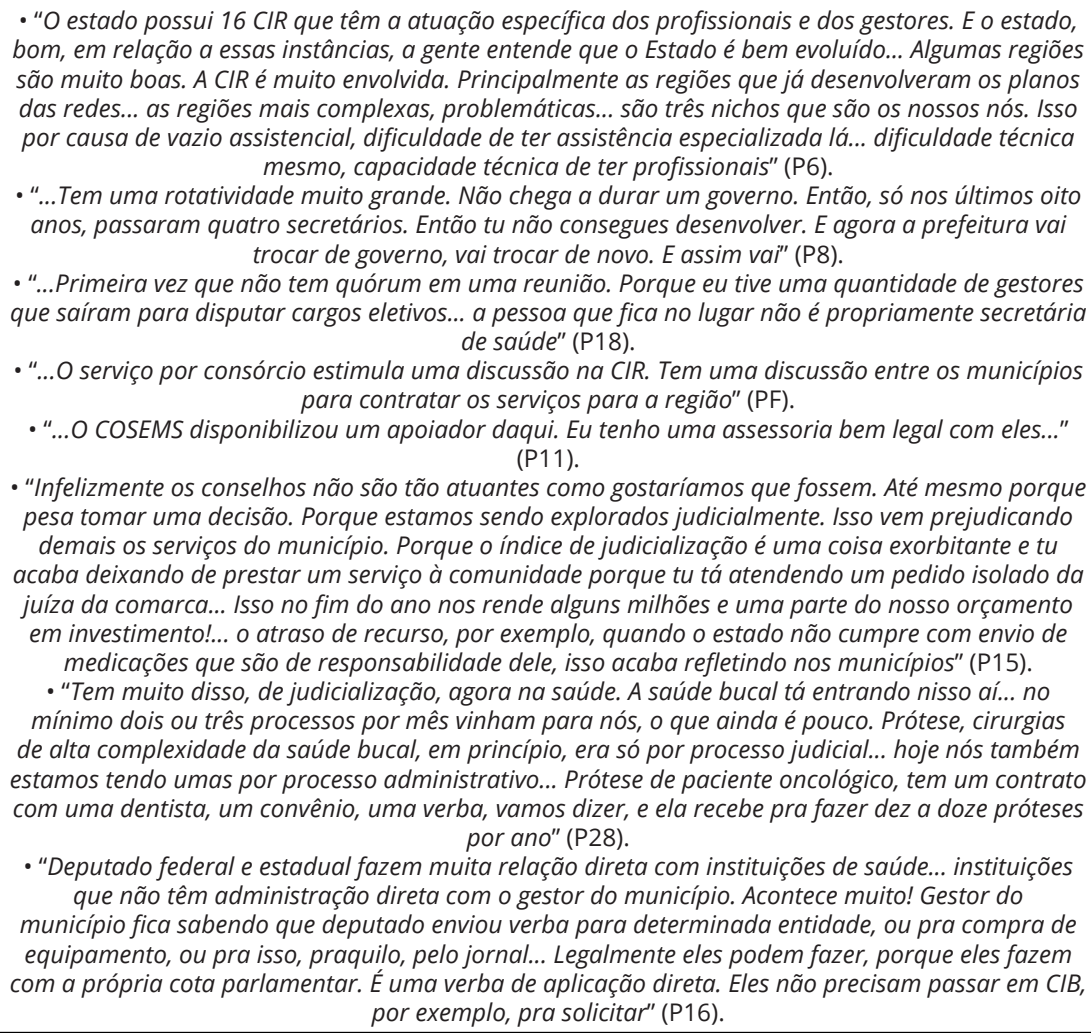 \\
\hline $\begin{array}{l}\text { Implementação de } \\
\text { redes temáticas de } \\
\text { saúde }\end{array}$ & $\begin{array}{c}\text { • Foco na estruturação } \\
\text { de redes temáticas } \\
\text { induzidas, } \\
\text { principalmente, por } \\
\text { incentivos financeiros } \\
\text { pelo nível federal e } \\
\text { conduzidas pelo estado. } \\
\text { • Estruturação de } \\
\text { câmaras técnicas para } \\
\text { negociação e tomada } \\
\text { de decisões quanto às } \\
\text { redes temáticas. } \\
\text { • Dúvidas sobre a } \\
\text { integração das redes } \\
\text { temáticas à rede de } \\
\text { atenção à saúde. }\end{array}$ & $\begin{array}{c}\text { - "Algumas vezes, se aquela região é muito articulada, então ela pressiona e consegue implantar } \\
\text { alguns serviços. Que é o caso, por exemplo, da Rede Cegonha que fez projetos regionalizados. Mas, } \\
\text { porque tinha o recurso do ministério... Então, alguma coisa avança, outras não. É diferente do caso } \\
\text { da Rede da Saúde Bucal, que não tem o incentivo federal como essas redes temáticas, então é uma } \\
\text { coisa que se constrói meio que no dia a dia" (P6). } \\
\text { - "Tem uma câmara técnica de gestão. Essa trabalha full time. Ela se reúne antes da reunião da CIR } \\
\text {... e tem as câmaras técnicas que a gente forma por um período. Agora a gente tá discutindo sobre } \\
\text { a urgência e emergência. Então, foram várias reuniões da câmara técnica até que a gente criasse o } \\
\text { plano regional de urgência e emergência" (P14). } \\
\text { - “...Tem melhorado muito, na questão da saúde bucal na atenção básica, mas a demanda de } \\
\text { média complexidade, ela é presente e precisa ser atendida. Então o município que não tem uma } \\
\text { referência, ele vai ter que comprar este serviço na iniciativa privada. Mas eu não saberia dizer, e não } \\
\text { tem ocorrido nestes quatro anos que estou na gestão, não tem ocorrido um momento de discussão } \\
\text { regional em relação a qual é a nossa demanda. Qual é nossa fila de espera? Estamos dando conta } \\
\text { ou não estamos? Que outras portas nós temos para isto? Porque às vezes nós temos laboratórios, ou } \\
\text { espaços de universidades, que na nossa região tem. Então, de que forma a gente pode usar isso? Eu } \\
\text { não vi, nesse tempo todo, uma discussão deste tipo" (P3). }\end{array}$ \\
\hline
\end{tabular}

(continua) 


\begin{tabular}{|c|c|c|}
\hline \multicolumn{3}{|c|}{$\begin{array}{l}\text { Categoria central } \\
\text { Influência das falhas da governança sobre a rede de atenção à saúde bucal: expansão errante de serviços por indução vertical e ratificação do } \\
\text { caráter periférico dado à saúde bucal. }\end{array}$} \\
\hline Categorias & Subcategorias & Exemplos de dados brutos \\
\hline $\begin{array}{l}\text { Planejamento } \\
\text { regional em saúde } \\
\text { imaturo e sob } \\
\text { efeito da crise } \\
\text { fiscal }\end{array}$ & $\begin{array}{c}\text { • Introdução do } \\
\text { planejamento } \\
\text { regional, com base } \\
\text { em discussões acerca } \\
\text { de demandas e } \\
\text { necessidades de saúde } \\
\text { dos municípios. } \\
\text { • Necessidade de focar } \\
\text { em discussões coletivas } \\
\text { e não apenas em apelos } \\
\text { individuais. } \\
\text { • Discussões em fórum } \\
\text { gestor centradas em } \\
\text { falta de recursos, } \\
\text { atrasos de repasses } \\
\text { dos governos } \\
\text { federal e estadual } \\
\text { e na interrupção de } \\
\text { programas. } \\
\text { • Fatores que } \\
\text { sobrecarregam } \\
\text { orçamentos municipais. }\end{array}$ & 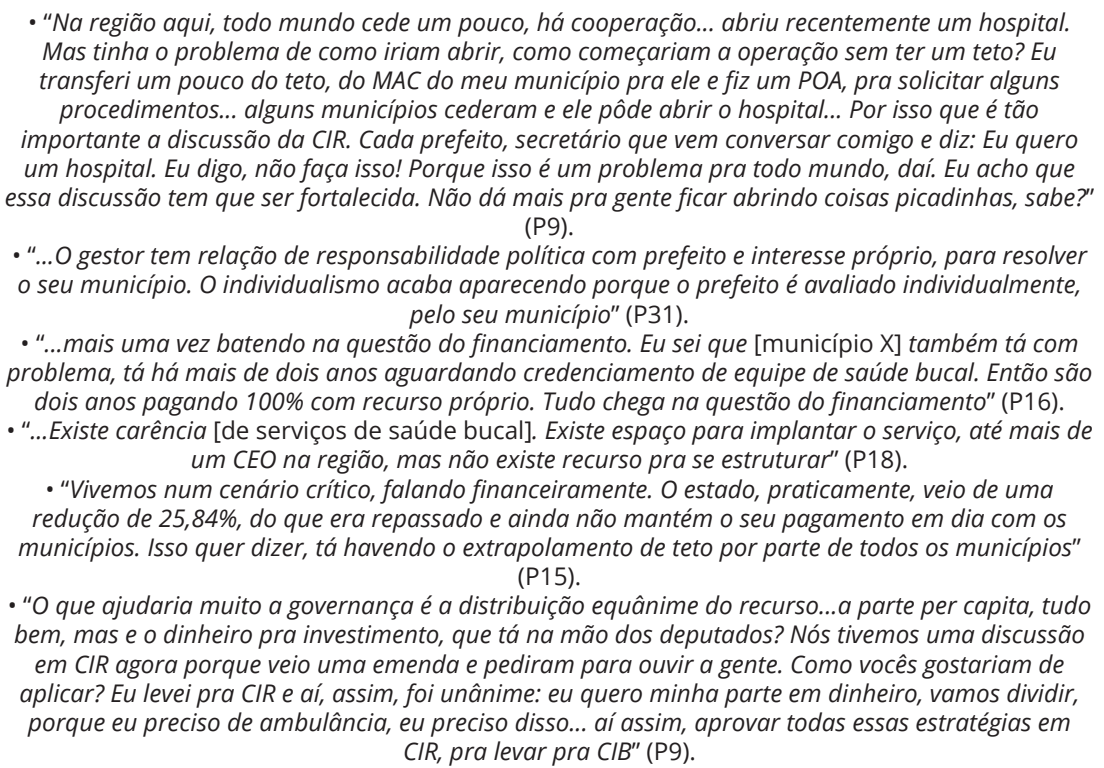 \\
\hline
\end{tabular}

CEO: Centro de Especialidades Odontológicas; CIB: Comissão Intergestores Bipartite; CIR: Comissão Intergestores Regional; COSEMS: Conselho de Secretarias Municipais de Saúde; MAC: limite financeiro da assistência de média e alta complexidade; POA: Plano Operativo Anual.

Para detalhar este resultado principal, a análise deu origem a três categorias identificadas como: Estabelecimento de estruturas formais de governança; Implementação de redes temáticas de saúde; e Planejamento regional em saúde imaturo e sob efeito da crise fiscal (Quadro 2).

A categoria Estabelecimento de estruturas formais de governança identifica as estruturas, mecanismos e instrumentos de governança formalmente estabelecidos e diretamente envolvidos com o processo de decisão para formulação/instituição da política de saúde no estado. Também sinaliza as estruturas de apoio e o impacto de agentes dos poderes executivo, legislativo e judiciário na execução desta política.

A categoria Implementação de redes temáticas de saúde demonstra, por meio dos participantes da pesquisa, ganhos para a governança com os incentivos, sobretudo os financeiros, à estruturação das redes temáticas - em especial as redes de Urgência e Emergência, Atenção à Saúde Materna e Infantil, Psicossocial e, mais recente e diretamente relacionada à atenção à saúde bucal, a Rede de Cuidados para a Pessoa com Deficiência. Tais redes estão sendo desenvolvidas em todas as regiões.

Houve algumas opiniões contrárias às subdivisões da rede de atenção à saúde por causa do prejuízo na conformação e integração de todas as redes temáticas na rede de atenção. No entanto, os entrevistados manifestaram que a estruturação de redes temáticas, induzidas e conduzidas por nível federal e estadual, respectivamente, estimulou a negociação e a tomada de decisões compartilhadas em CIR. Paralelamente, incentivou a instituição da realização de diagnóstico das estruturas e necessidades existentes nas regiões e o estabelecimento de diretrizes e protocolos que permitem a construção e o monitoramento dessas redes. 
A categoria Planejamento regional em saúde imaturo e sob efeito da crise fiscal revela que, com a consolidação do fórum gestor em nível regional, as discussões acerca da pertinência e atualização dos mapas de saúde estaduais foram iniciadas, primeiramente seguindo as redes temáticas implantadas. Também desencadeou a prática de acolher e valorizar as demandas e necessidades em saúde dos municípios. Ocorreram incentivos via SES e Conselho de Secretarias Municipais de Saúde (COSEMS), por intermédio de estímulos à capacitação dos gestores e sua efetiva participação em comissões intergestoras, para que fosse concretizado o planejamento regional. Contudo, reiteradamente os participantes apontaram a necessidade de superar discussões que focam em interesses individuais e concentrar esforços no coletivo.

O modelo representa também a necessidade de resolução das fragilidades identificadas nos atributos de governança, destacados a seguir, a fim de que se possa alcançar um planejamento estadual de base regional.

O Quadro 3 apresenta as falhas identificadas nos atributos de governança: Transparência, Accountability, Participação, Integridade e Capacidade Política que contribuem para identificar o padrão de governança existente no sistema de saúde sob análise, bem como para compreender a influência da governança identificada sobre o tipo e as consequências da tomada de decisão.

Os resultados desvelam fragilidades que dificultam a estruturação da atenção à saúde bucal em perspectiva de rede regionalizada de atenção. A insuficiência, alta rotatividade e falta de capacidade técnica ou experiência de gestores impactam na escolha de políticas, na continuidade das ações e na integração entre as áreas gestoras, bem como no apoio técnico às regiões de saúde.

Os resultados apontam que a atual discussão sobre implantação do complexo regulador ambulatorial pode influenciar na estruturação de uma rede regionalizada de atenção à saúde bucal. Há estímulos para envolvimento de coordenadores da atenção à saúde bucal nas discussões para iniciar a identificação dos serviços, especialmente os relacionados à Rede da Pessoa com Deficiência, e para construção de fluxos e protocolos para regulação. Apesar de ser reconhecida a grande expansão de serviços de atenção básica também relacionados à saúde bucal, a escassez de serviços especializados é constatada da mesma forma. A ausência de protocolos, diretrizes e regulação via Central de Regulação Ambulatorial são falhas na Transparência, impactam nas relações de Accountability e foram apontadas como razões para a dificuldade de integração entre as ações de saúde bucal e provisão de serviços odontológicos.

O modelo destaca a expansão dos serviços em atenção primária e secundária, fato que muitos participantes caracterizaram como "programa do Ministério da Saúde", portanto, verticalmente induzida. Ela é impulsionada pela SES pela identificação de vazios assistenciais de serviços especializados e com orientações para adesão à Estratégia Saúde da Família (ESF). Porém, constata-se que o papel da SES se torna limitado, com problemas de Capacidade Política e Integridade na governança estudada, pela falta de recursos humanos em quantidade e capacidade técnica suficiente, tanto na estrutura central, quanto nas GERSA, que estimulariam o planejamento regionalizado.

Por outro lado, o modelo revela a ausência de integração entre gestores da rede de atenção à saúde bucal e demais áreas, bem como a falta de protagonismo no desenvolvimento da Política Estadual de Saúde Bucal, a partir da identificação das estruturas estabelecidas e das necessidades da população. A ausência de tal política extrapola a constatação da limitada oferta de serviços especializados. É também percebida quando não reconhecem a saúde bucal como prioridade em saúde pública - por parecer não ter grande impacto na sociedade pela priorização populacional e demanda assistencial e aparentar pouco apelo político-eleitoral. Ademais, é considerado por muitos gestores um serviço oneroso para estruturação e, sobretudo, para seu custeio.

Esse caráter periférico também pode ser constatado nas soluções improvisadas que afetam os atributos de Capacidade Política, Accountability e Integridade, tais como mecanismos informais para estruturar a gestão e a regulação dos serviços de saúde bucal no momento em que os profissionais exercem funções de gestão e assistência concomitantemente, dividindo sua carga horária de trabalho e, por vezes, não possuindo capacidade técnico-gerencial, como relatado pelos entrevistados. Adiciona-se o isolamento ainda persistente dos profissionais de saúde bucal em relação às demais áreas da saúde. Também foi relatada a inserção superficial do tema saúde bucal nos planos de saúde, ratificando a fragilidade dos instrumentos de governança e a ausência de dados/informações em saúde bucal. 
Quadro 3

Potenciais falhas encontradas nos atributos de governança na rede de atenção à saúde bucal. Santa Catarina, Brasil, 2018.

\begin{tabular}{|c|c|}
\hline Atributo & Potenciais falhas encontradas \\
\hline Transparência & $\begin{array}{c}\text { - Limitações no compartilhamento de informações sobre tomada de decisão e formulação de políticas entre instâncias } \\
\text { gestoras e conselhos de saúde. } \\
\text { - Fragilidades em sistemas de informação, por vezes sem interoperabilidade, com imprecisões de dados que afetam o } \\
\text { desenvolvimento de instrumentos como o Mapa da Saúde, assim como o compartilhamento de dados entre todos os } \\
\text { stakeholders. } \\
\text { • Dificuldades na entrega de relatórios de gestão. } \\
\text { - Ausência de dados epidemiológicos. }\end{array}$ \\
\hline Accountability & $\begin{array}{r}\text { - Presença marcante e constante de influências políticas com interesses eleitorais que prejudicam a tomada de decisão } \\
\text { em instâncias coletivas de gestão e participação social. } \\
\text { - Ausência de política de saúde robusta, planejada com base nas necessidades da população, e de longo prazo que } \\
\text { evite interferências políticas partidárias e/ou interesses privados, bem como diretrizes e protocolos que estabeleçam } \\
\text { responsabilidades de cada setor/unidade. } \\
\text { - Resistência e ausência de acordo entre as partes para instituição do COAP como mecanismo de contratualidade, } \\
\text { estando sob forte influência do ajuste fiscal. } \\
\text { - Mecanismos de financiamento fragilizados por conta do corte de recursos, atrasos no repasse e devido à forma de } \\
\text { financiamento por adesão a programas que também sofreram cortes. } \\
\text { - Processos de judicialização na saúde que acarretam em sobrecarga de orçamentos e dificuldade de financiamento. } \\
\text { - Regulação ambulatorial incipiente em algumas regiões: limitações quanto à contratação de profissionais, carência de } \\
\text { fluxos definidos e serviços especializados, assim como há conflitos para regulação de consórcios. }\end{array}$ \\
\hline Participação & $\begin{array}{c}\text { • Fragilidades na legitimidade e representação dos conselhos pela dificuldade em manter adesão de conselheiros, } \\
\text { sua paridade, bem como o reconhecimento de suas funções para que ultrapasse o caráter meramente burocrático e } \\
\text { homologatório, e participe ativamente da governança. } \\
\text { - Persistente manutenção de gestores municipais como presidentes dos conselhos e relatos de influências político- } \\
\text { partidárias em pautas do conselho. } \\
\text { - Persistente rotatividade de gestores e técnicos de saúde por conta de interferência político-eleitoral em todos os níveis. } \\
\text { Prejudica a participação de representantes dos municípios e técnicos da SES nas comissões. } \\
\text { - Nomeação de gestores com qualificação comprometida ou sem experiência, comprometendo planejamento e } \\
\text { regionalização. } \\
\text { Grande diversidade na capacidade de atuação das CIR. }\end{array}$ \\
\hline Integridade & $\begin{array}{l}\text { - Falha de integridade por nomeações de gestores em saúde ocorrerem de acordo com política eleitoral e por curto } \\
\text { prazo, acarretando em alta rotatividade de profissionais. } \\
\text { - Ausência de integração entre áreas gestoras da SES. } \\
\text { - Desencontro de informações entre os níveis de governo e dificuldade de reconhecimento claro do papel e } \\
\text { responsabilidade das estruturas de governança. }\end{array}$ \\
\hline Capacidade Política & $\begin{array}{c}\text { - Problemas quanto à capacidade técnica e experiência de gestores e conselheiros de saúde em todos os níveis, } \\
\text { acarretando, por vezes, uma relação de dependência entre as estruturas menos capacitadas e as mais desenvolvidas. } \\
\text { - Avanços modestos quanto à oferta de capacitação de gestores e conselheiros de saúde por meio de SES, COSEMS e } \\
\text { alguns municípios, porém ainda há grande necessidade de capacitação por causa da rotatividade. } \\
\text { - Necessidade de aperfeiçoar método de capacitação de gestores e conselheiros devido aos custos para deslocamento. } \\
\text { - Interrupção do trabalho de câmaras técnicas em razão da carência de RH e rotatividade de gestores, prejudicando as } \\
\text { discussões técnicas sobre a organização e estruturação das redes temáticas. } \\
\text { - Foco em construção de redes induzidas e com incentivos federais e estaduais, com carência de grupos técnicos para } \\
\text { resolver demandas locais/regionais. }\end{array}$ \\
\hline
\end{tabular}

CIR: Comissão Intergestores Regional; COAP: Contrato Organizativo da Ação Pública; COSEMS: Conselho de Secretarias Municipais de Saúde; SES: Secretaria Estadual de Saúde.

Os participantes apontam que, mesmo em locais com oferta de serviços de saúde bucal especializados e regionalizados, há escassez de discussão entre os municípios envolvidos, gerando sobrecarga para os municípios-sede.

As pautas discutidas em CIR e CIB concentram-se em explorar as redes temáticas instituídas por portaria ministerial, a implantação do complexo regulador e, especialmente, sobre a dificuldade de financiamento em saúde e sobrecarga dos municípios. As poucas discussões realizadas em CIR sobre estruturação da rede de saúde bucal se relacionam predominantemente à dificuldade de custeio dos serviços regionalizados. O corte na habilitação de serviços de saúde bucal na ESF, ocasionado pelo atual contingenciamento nas transferências federais, também foi apontado como fator que sobrecarrega o orçamento municipal. 
A atual experiência com serviços especializados de saúde bucal, por meio de consórcios intermunicipais de saúde, foi apontada como satisfatória e, assim como citado para os demais serviços especializados de saúde em geral, poderiam ser uma alternativa para a organização dos municípios interessados, desde que exista comprometimento de todos participantes para contratação e/ou manutenção do serviço.

Ainda que se reconheçam as limitações na estruturação da atenção à saúde bucal, regionalmente e no estado, a rede de saúde é percebida, paradoxalmente, pelos participantes como bem estruturada. Foi apontada a necessidade de conscientização dos gestores, em todos os níveis, sobre a relevância e a extensão dos agravos à saúde bucal como problema relevante em saúde pública, bem como sobre a atual situação epidemiológica das diferentes regiões do Estado de Santa Catarina.

Foi reconhecido que a instituição de uma rede temática para saúde bucal e a formação de câmara técnica regional e estadual poderiam contribuir para a estruturação formal do cuidado e maior visibilidade para os problemas da saúde bucal na população. Tal mecanismo de governança, segundo os entrevistados e com base em experiências de outras redes temáticas, permitiria discussões aprimoradas com gestores e técnicos comprometidos com a instituição da rede.

\section{Discussão}

O modelo teórico emergente neste estudo dá conta de compreender o caráter da governança empreendida atualmente na rede regionalizada de atenção à saúde em Santa Catarina e seus óbices que influenciam a tomada de decisão e proposição de políticas ${ }^{3}$. Foi possível reconhecer as estruturas atuantes nesse processo e seu caráter multinível - por se constituir em um sistema federativo e com uma estratégia de regionalização das ações e serviços de saúde pela organização das redes temáticas de atenção $3,9,28$.

O estudo pode identificar as instâncias que procuram alinhar as diferentes agendas, instituir o compartilhamento de poder e da tomada de decisão, bem como aquelas que almejam produzir resultados coerentes e com objetivos comuns voltados para a instituição de um sistema de saúde público universal, de qualidade e sustentável ${ }^{3}$.

As relações conflituosas e as repetidas trocas de poder foram elementos presentes. Entretanto, ressalta-se que o conflito, enquanto arena de disputa, e as mudanças político-eleitorais influenciando negativamente a governança em saúde não são exclusividade do estado de Santa Catarina. A consolidação da regionalização e a construção de redes de atenção à saúde mostram-se como desafios para governança em diversos estudos 6,9,10,21. O protagonismo do espaço regional e a priorização de um planejamento regionalizado precisam ser tomados como objetivo dos diversos stakeholders, incluindo chefes do executivo, legisladores, gestores de saúde, profissionais e representantes da sociedade civil, de forma a produzir planos com base nas diretrizes do SUS em curto, médio e longo prazos 10. O perfil dos gestores em saúde, responsáveis tanto pela coordenação regional, gestão municipal, estadual e coordenação de serviços odontológicos, é replicado em nível ampliado ${ }^{29}$, sendo em sua maioria homens mais maduros e que possuem nível superior.

Os óbices nos atributos Transparência, Accountability, Participação, Integridade e Capacidade Política tornam mais difícil a tomada de decisão coletiva que leve em conta os interesses de todos os envolvidos 3 .

As falhas em Transparência implicam menor confiança no processo de tomada de decisão. Geram dúvidas sobre como as decisões e as formulações são realizadas, bem como dificultam a ação de agentes internos e externos na contribuição e acompanhamento das políticas ${ }^{3}$. De mais a mais, a ausência e/ou limitações em instrumentos que definam claramente as responsabilidades e o dever de dar contas entre atores e estruturas de governança comprometem as relações de Accountability ${ }^{3}$. Tal debilidade nos instrumentos disponíveis e necessidade de desenvolvimento de ferramentas capazes de coordenar e regular todos os serviços, próprios ou por consórcios, e contribuir com o planejamento regional são alguns dos principais desafios à governança 11.

A ausência de continuidade de políticas e estabilidade de profissionais afeta a Integridade da governança. Assim como, esta é afetada pela dificuldade que possuem em reconhecer claramente as funções de cada ente ${ }^{3}$. Essas falhas influenciam na falta de profissionais que advoguem pela estrutu- 
ração da atenção à saúde bucal no estado, em perspectiva regionalizada, e no isolamento dos setores designados para gestão dos serviços de saúde bucal. Todavia, as funções e o papel de cada nível de governo se sobrepõem por conta, também, do caráter federativo. Há uma mistura entre competências comuns e competências concorrentes e/ou específicas de cada esfera. A formulação de políticas é competência comum das três esferas e tem sido compartilhada atualmente por meio das Comissões Intergestores 10,30,31.

É comum argumentar-se que o crescimento exponencial dos custos do sistema de saúde pela situação de tripla carga de doenças 32 , a crescente judicialização do direito à saúde em todo o país 5,33,34, as atuais políticas associadas à crise fiscal, que afetam a demanda por serviços públicos e acarretam em maiores cortes nos recursos destinados à saúde 33,34,35, exacerbam o problema de subfinanciamento do SUS 34,35 e o tornam a principal pauta de discussão nas instâncias intergestoras. Assim sendo, há uma pressão sobre o sistema e sobre os gestores de saúde ${ }^{3}$, particularmente sobre gestores municipais que arcam com maior comprometimento do orçamento 5 . Dessa forma, ela se torna uma questão urgente para o debate locorregional, afeta a Integridade do sistema e contribui para a marginalização das demais discussões sobre organização da rede regionalizada de atenção ou o estabelecimento de novas políticas 3,6 .

A relação entre os poderes executivo, legislativo e judiciário, vista pelo ângulo do financiamento do SUS 33, foi evidenciada pelos participantes do estudo que apontaram grave falta de coordenação capaz de levar o sistema ao colapso 33,35. Além disso, as relações intergovernamentais, o compartilhamento de poder, a dificuldade de contratualidade entre os entes e os demais problemas evidenciados nos atributos estudados conduzem a um cenário que causa desafios para a governança, especialmente na eleição de prioridades e realização do planejamento de forma regionalizada 3,35.

Esse cenário perpetua o caráter periférico dado para estruturação da atenção à saúde bucal em rede de atenção com base em discussões regionalizadas e nas necessidades da população. Estudo anterior, realizado no âmbito da CIB catarinense, também constatou esse padrão 21. Esse posicionamento resulta de deficiências históricas relacionadas ao isolamento da profissão odontológica, bem como da persistente percepção social do direito a saúde bucal como secundário e da ausência de voz e influência política dos estratos sociais mais baixos, justamente os que possuem maior carga da doença 16,21.

Verificou-se um processo de tomada de decisão verticalizado e induzido por financiamento federal, que se poderia chamar de "expansão errante" dos serviços. Esse termo foi cunhado por Reis et al. 10 ao relatar a inexistência de planejamento regional para a rede de serviços de saúde em geral, com decisões tomadas por um ente isolado, seguidas pelos demais que demandam, desarticuladamente, por recursos do ente federal para continuidade das ações e serviços.

Como constado no presente estudo, a atenção à saúde bucal não é reconhecida como prioridade em saúde pública 15 . Atualmente, esse é um problema advindo das fragilidades da governança, das discussões concentradas em problemas de financiamento, e, ainda, da carência de recursos humanos qualificados e de uma política de saúde bucal estruturada no âmbito estadual. Os agravos bucais apresentam um menor nível de engajamento social e interesse público em todo mundo 36,37. Apesar de esses agravos, crônicos em geral, raramente apresentarem um risco à vida do indivíduo, eles apresentam profundo impacto sobre a qualidade de vida das pessoas. O ônus social e econômico gerado pelas doenças bucais deve ser destacado para buscar maior engajamento social e inclusão efetiva na agenda política. Portanto, precisa criar maior conscientização pública e ampliar a valorização da saúde bucal, de modo a fortalecer a pressão social sobre as estruturas e mecanismos de governança 36,37.

Há evidências na literatura científica sobre a importância dos agravos bucais para a saúde pública em todo o mundo 13,36,37, assim como é reconhecido seu padrão de determinação social 36,37. Estudos brasileiros 3,18,38 demonstram que, apesar de terem ocorrido avanços consideráveis no perfil epidemiológico da população, notadamente em crianças e adolescentes, a perda dentária entre idosos é extremamente alta e o país ainda revela uma grande desigualdade regional e social nas condições de saúde bucal em todas as idades e também no acesso e uso de serviços odontológicos. Ademais, apesar de haver estudos demonstrando a ampliação de serviços públicos em odontologia 16 e uma tendência redistributiva da oportunidade de acesso aos serviços de atenção básica, considerada pró-equidade 39 , os desafios para superar a desigualdade em saúde bucal e no uso dos serviços permanecem 18,37,38.

Apesar da inclusão da saúde bucal na agenda nacional de prioridades de saúde pública, a partir da PNSB 16, que traça diretrizes para reorganização do cuidado à saúde bucal em todos os níveis, o desa- 
fio da implantação dessa política e das ações e serviços requerem melhorias na governança vigente 40 . A incorporação e resgate da atenção à saúde bucal em rede regionalizada requererá a superação das deficiências apontadas neste estudo.

Este estudo apresenta limitações inerentes ao seu desenho de pesquisa e não pretende realizar generalizações. Os resultados oferecem interpretações de um fenômeno que ocorre na realidade investigada, mas a teoria fundamentada nos dados que foi utilizada pode ser aplicada em outros contextos 22,23 . Os resultados também se referem a um contexto histórico singular, refletindo os processos de gestão que aconteceram e estão acontecendo em um determinado momento. O processo de pesquisa em uma teoria fundamentada compreende um espaço de tempo extenso. Nesse caso específico, compreendeu mudanças relevantes na implementação da PNSB que se procurou incorporar ao modelo.

Sugere-se a realização de estudos que apontem estratégias para lidar com as falhas de governança aqui identificadas, as quais repercutirão positivamente na estruturação da atenção à saúde bucal no país numa perspectiva regionalizada. Analisar e aplicar essas estratégias será fundamental para o enfrentamento da implantação errante dos serviços de saúde bucal, da mera reprodução da PNSB sem observar as realidades e necessidades regionais, e para a valorização e inclusão da saúde bucal em todas as políticas.

De modo construtivo, este estudo aponta que a estruturação da atenção à saúde bucal na perspectiva de rede regionalizada poderia desde logo ser objeto de discussão em câmaras técnicas em cada uma das CIR 21,32. As câmaras técnicas deveriam ser formadas por profissionais da saúde e gestores estratégicos, com conhecimento e espírito de advocacy, com intuito de propugnar um novo protagonismo da atenção à saúde bucal na rede e se beneficiar das vantagens deste mecanismo de governança.

\section{Colaboradores}

H. Godoi contribuiu com a concepção do estudo, coleta, análise e interpretação dos dados, redação e revisão do artigo e aprovação da versão final. R. G. Castro contribuiu com a revisão crítica relevante do conteúdo intelectual e aprovação da versão final. J. L. G. Santos contribuiu com revisão do método empregado, redação do artigo, revisão crítica relevante do conteúdo intelectual e aprovação da versão final. S. J. Moyses contribuiu com a redação do artigo, revisão crítica relevante do conteúdo intelectual e aprovação da versão final. A. L. S. F. Mello contribuiu com a concepção do estudo, análise e interpretação dos dados, redação do artigo, revisão crítica relevante do conteúdo intelectual e aprovação da versão final.

\section{Informações adicionais}

ORCID: Heloisa Godoi (0000-0002-7684-7692);

Renata Goulart Castro (0000-0002-2951-5775); José Luís Guedes dos Santos (0000-0003-31868286); Samuel Jorge Moyses (0000-0003-30756397); Ana Lúcia Schaefer Ferreira de Mello (00000001-9591-7361). 


\section{Referências}

1. Pyone T, Smith H, van den Broek N. Frameworks to assess health systems governance: a systematic review. Health Policy Plan 2017; 32:710-22.

2. Hufty M. Gobernanza en salud pública: hacia un marco analítico. Rev Salud Pública 2010; 12:39-61.

3. Greer SL, Wismar M, Figueras J. Strengthening health system governance. Better policies, stronger performance. New York: Open University Press; 2016.

4. Almeida LL. O significado de governança para os gestores estaduais do Sistema Único de Saúde. Brasília: Universidade de Brasília; 2013.

5. Shimizu HE, Cruz MS, Bretas N, Schierholt SR, Ramalho WM, Ramos MC, et al. The prominent role of Councils of Municipal Health Secretariats in the regional governance process. Ciênc Saúde Colet 2017; 22:1131-40.

6. Santos AM, Giovanella L. Regional governance: strategies and disputes in health region management. Rev Saúde Pública 2014; 48:622-31.

7. Fleury S, Ouverney ALM, Kronemberger TS, Zani FB. Governança local no sistema descentralizado de saúde no Brasil. Rev Panam Salud Pública 2010; 26:446-55.

8. Jakubowski E, Saltman RB. The changing national role in health system governance. Geneva: World Health Organization; 2013.

9. Bretas N, Shimizu HE. Theoretical reflections on governance in health regions. Ciênc Saúde Colet 2017; 22:1085-95.

10. Reis AACD, Sóter APM, Furtado LAC, Pereira SSDS. Thoughts on the development of active regional public health systems. Ciênc Saúde Colet 2017; 22:1045-54.

11. Mello GA, Pereira APCM, Uchimura LYT, Iozzi FL, Demarzo MMP, Viana ALD. A systematic review of the process of regionalization of Brazil's Unified Health System, SUS. Ciênc Saúde Colet 2017; 22:1291-310.

12. Viana ALA, Lima LD, Ferreira MP. Structural conditions for regionalization in health care: typology of Regional Management Boards. Ciênc Saúde Colet 2010; 15:2317-26.

13. Kassebaum NJ, Smith AGC, Bernabé E, Fleming TD, Reynolds AE, Vos T, et al. Global, regional, and national prevalence, incidence, and disability-adjusted life years for oral conditions for 195 countries, 1990-2015: a systematic analysis for the global burden of diseases, injuries, and risk factors. J Dent Res 2017; 96:380-7.

14. Dye BA. The global burden of oral disease: research and public health significance. J Dent Res 2017; 96:361-3.

15. Costa JSD, Victora CG. O que é "um problema de saúde pública”? Rev Bras Epidemiol 2006; 9:144-6.

16. Pucca GA, Gabriel M, de Araujo ME, de Almeida FCS. Ten years of a National Oral Health Policy in Brazil. J Dent Res 2015; 94:1333-7.
17. Godoi H, Mello AL, Caetano JC. Rede de atenção à saúde bucal: organização em municípios de grande porte de Santa Catarina, Brasil. Cad Saúde Pública 2014; 30:318-32.

18. Peres KG, Peres MA, Boing AF, Bertoldi AD, Bastos JL, Barros AJ. Reduction of social inequalities in utilization of dental care in Brazil from 1998 to 2008. Rev Saúde Pública 2012; 46:250-8.

19. Peres MA, Fernandes LS, Peres KG. Inequality of water fluoridation in Southern Brazil: the inverse equity hypothesis revisited. Soc Sci Med 2004; 58:1181-9.

20. Mello ALSF, Andrade SR, Moysés SJ, Erdmann AL. Saúde bucal na rede de atenção e processo de regionalização. Ciênc Saúde Colet 2014; 19:205-14.

21. Godoi H, Andrade SR, Mello ALSF. Rede regionalizada de atenção à saúde no Estado de Santa Catarina, Brasil, entre 2011 e 2015: sistema de governança e a atenção à saúde bucal. Cad Saúde Pública 2017; 33:e00133516.

22. Glaser B. The grounded theory perspective II: description's remodelling of grounded theory methodology. Mill Valley: Sociology Press; 2003.

23. Holton JA, Walsh I. Classic grounded theory: applications with qualitative and quantitative data. Los Angeles: SAGE; 2017.

24. Secretaria de Estado da Saúde. Linha de cuidado de saúde bucal. Florianópolis: Secretaria de Estado da Saúde; 2019.

25. Glaser B, Strauss AL. The discovery of Grounded Theory: strategies for qualitative research. New York: Aldine de Gruyter; 1967.

26. Fontanella BJB, Ricas J, Turato ER. Amostragem por saturação em pesquisas qualitativas em saúde: contribuições teóricas. Cad Saúde Pública 2008; 24:17-27.

27. Brasil. Decreto no 7.508 , de 28 de junho de 2011. Regulamenta a Lei no 8.080, de 19 de setembro de 1990, para dispor sobre a organização do Sistema Único de Saúde - SUS, o planejamento da saúde, a assistência à saúde e a articulação interfederativa, e dá outras providências. Diário Oficial da União 2011; 29 jun.

28. Lima LD, Scatena JGH, Albuquerque MV, Oliveira RAD, Martinelli NL, Pereira AMM. Arranjos de governança da assistência especializada nas regiões de saúde do Brasil. Rev Bras Saúde Matern Infant 2017; 17 Suppl 1:S12133.

29. Moreira MR, Ribeiro JM, Ouverney AM. Political obstacles to regionalization of the SUS: perceptions of Municipal Health Secretaries with seat in the Bipartite Interagency Commissions. Ciênc Saúde Colet 2017; 22:1097108. 
30. Lima LD, Machado CV, Baptista TWF. O papel das três esferas de governo na gestão do SUS. In: Kuschnir RO, organizadora. Gestão de Redes de Atenção à Saúde. v. 1. Rio de Janeiro: Escola Nacional de Saúde Pública Sergio Arouca, Fundação Oswaldo Cruz; 2014. p. 59-92.

31. Machado CV, Lima LD, Viana ALA, Oliveira RG, Iozzi FL, Albuquerque MV, et al. Federalism and health policy: the intergovernmental committees in Brazil. Rev Saúde Pública 2014; 48:642-50.

32. Mendes EV. As redes de atenção à saúde. Brasília: Organização Pan-Americana da Saúde; 2011.

33. Santos AO, Delduque MC, Alves SMC. Os três poderes do Estado e o financiamento do SUS: o ano de 2015. Cad Saúde Pública 2016; 32:e00194815.

34. Padilha A, Oliveira DC, Alves TA, Campos GWS. Crise no Brasil e impactos na frágil governança regional e federativa da política de saúde. Ciênc Saúde Colet 2019; 24:4509-18.

35. Mendes A. The Brazilian public health in a universe "worldless": the austerity of the Constitutional Amendment Proposition 241/2016. Cad Saúde Pública 2016; 32:e00188916.
36. Watt RG. Addressing oral health inequalities: where do we stand? In: Peres MA, Watt RG, editors. Policy solutions for oral health inequalities. Adelaide: Australian Research Centre for Population Oral Health; 2017. p. 1-4. (International Centre for Oral Health Inequalities Research \& Policy (ICOHIRP), monograph 2).

37. Watt RG, Daly B, Allison P, Macpherson LMD, Venturelli R, Listl S, et al. Ending the neglect of global oral health: time for radical action. Lancet 2019; 394: 261-72.

38. Chaves SCL, Almeida AMFL, Rossi TRA, Santana SF, Barros SG, Santos CML. Oral health policy in Brazil between 2003 and 2014: scenarios, proposals, actions, and outcomes. Ciênc Saúde Colet 2017; 22:1791-803.

39. Fernandes JK, Pinho JR, Queiroz RC, Thomaz EB. Evaluation of oral health indicators in Brazil: a trend towards equity in dental care? Cad Saúde Pública 2016; 32:e00021115.

40. Soares CL. Constructing public oral health policies in Brazil: issues for reflection. Braz Oral Res 2012; 26 Suppl 1:94-102. 


\section{Abstract}

The study aimed to analyze public governance in the regionalized healthcare network in the state of Santa Catarina, Brazil, and its influence on decision-making in the organization of oral healthcare. This was an exploratory analytical study with a qualitative approach, with the methodology based on Grounded Theory. Thirty in-depth interviews were held with managers from the Regional Inter-Managers Commissions and Bipartite Commissions (CIR and CIB, respectively, in Portuguese), under the State Health Department, representatives from the State Health Councils, and administrators of specialized oral healthcare services, in two sample groups, in addition to nonparticipant observation in CIB meetings. After comparative data analysis, the theoretical model was formulated in which the central category was expressed in the title Influence of Failures in Governance on the Oral Healthcare Network: The Erratic Expansion of Services by Vertical Induction and Confirmation of the Peripheral Status Assigned to Oral Health. The governance practiced in the Santa Catarina State Health System displays well-established formal support structures, such as the consolidation of the CIR. However, weaknesses were identified that reveal problems in the governance systems' legitimacy. The situation perpetuates the peripheral status assigned to the structuring of oral healthcare in network format and the erratic expansion of services, characterized by vertical induction and without contextualized recognition of oral health problems as guidance for planning the services. There is a need for greater public awareness and the inclusion of professionals and managers that can advocate for the importance of oral health as a major priority in public health policies.

Health Services Administration; Regional Health Planning; Oral Health; Health Sector Stewardship and Governance

\section{Resumen}

El objetivo del trabajo fue analizar la gobernanza pública emprendida en la red regionalizada de atención en salud en Santa Catarina, Brasil, y su influencia sobre la toma de decisiones respecto a la estructuración de la atención en la salud bucal. Se trata de un estudio exploratorio-analitico, con enfoque cualitativo, y apoyado metodológicamente en la Teoría Fundamentada en Datos. Se realizaron 30 entrevistas en profundidad, con gestores de las Comisiones Intergestoras Regional (CIR) y Bipartita (CIB), de la Secretaría Provincial de Salud, representantes del Consejo Provincial de Salud y gestores de servicios especializados en salud bucal, en dos grupos de muestras; además de la observación no participante en reuniones de la CIB. Tras el análisis comparativo de los datos, se formuló un modelo teórico, cuya categoría central se expresó bajo el título Influencia de los Fallos de la Gobernanza sobre la Red de Atención en Salud Bucal: La Expansión Errante de Servicios por Inducción Vertical y Ratificación del Carácter Periférico Otorgado a la Salud Bucal. La gobernanza emprendida en el sistema de salud catarinense presenta estructuras formales $y$ de apoyo bien establecidas, como la consolidación de las CIR. No obstante, se identificaron fragilidades, que revelan problemas en la legitimidad del sistema de gobernanza. Se perpetúa su posición periférica, debido a la estructuración en red de la atención en salud bucal y la expansión errante de sus servicios, caracterizada por la inducción vertical, y sin el reconocimiento contextualizado de los problemas en salud bucal, como guía para la planificación de los servicios. Existe la necesidad de una mayor concienciación pública, así como la inclusión de profesionales y gestores con espíritu de advocacy para que propugnen un nuevo protagonismo de la atención en salud bucal dentro de las políticas públicas de salud.

Administración de los Servicios de Salud; Regionalización; Salud Bucal; Rectoría y Gobernanza del Sector de Salud
Recebido em 28/Set/2019

Versão final reapresentada em 18/Fev/2020

Aprovado em 17/Abr/2020 